\section{PROTECT YOUR PATIENTS' SMILES WITH QUALITY SPORTS GUARDS}

Available in a wide range of colours, Sparkle Dental Labs mouthguards are custom-made in the UK to ensure a perfect fit. With sophisticated and modern materials, protection and durability is increased. These modern and sophisticated mouthguards are specifically designed to fit comfortably, allowing easy speech, swallowing and breathing while achieving ultimate protection against hard and soft object collisions.

Furthermore, the service at Sparkle Dental Labs is second to none, and with all manufacturing performed expertly in the state-of-the-art dental labs, you can rest assured that products are of a high quality and conform to exacting British standards. With free collection and delivery, fast turnaround of orders, and excellent customer service, mouthguards can be remodelled quickly and efficiently so patients do not have to wait longer than necessary for changes to be made.

Ensure your patients stay adequately protected when participating in any sporting event with a quality mouthguard from Sparkle Dental Labs.

For more details about Sparkle Dental Labs, call 0800138 6255, email customerservice@sparkledentallabs.com or visit www.sparkledentallabs.com.

\section{THE SIGNIFICANCE OF BREATH}

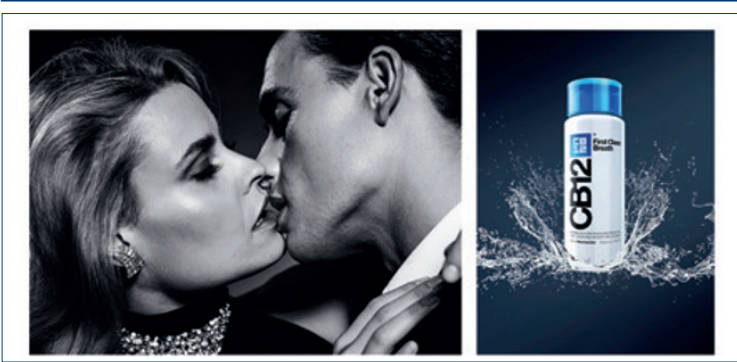

From Cicero's 'As long as I breathe, I hope' to Shakespeare's 'foul breath is noisome' the importance of breath has fascinated writers through the ages.

Around one in four adults in the UK are thought to regularly suffer from bad breath. While it may be the result or indication of an underlying medical condition, in most cases the problem originates in the oral cavity with malodorous gases known as volatile sulphur compounds (VSCs).

Bad breath can be worrying and embarrassing for sufferers. Fortunately, CB12 neutralises the VSCs that cause bad breath, rather than just masking the smell. Its unique formula is effective for up to 12 hours and helps to maintain fresh breath all day.

Helping to relieve bad breath can make a big difference to sufferers. Shakespeare, as always, puts it well: 'For this relief much thanks'!

For more information about CB12 visit www.cb12. co.uk or to order contact DHB oral healthcare on 08456017086 or www.dhb.co.uk

\title{
TOP QUALITY TRAINING FOR CONSERVATIVE DENTISTRY
}

Sarah Farrier is the Principal Dentist of Arden House Dental in Cheltenham and she undertook the hands-on training course for the Inman Aligner in London in July 2014.

She said: 'I took the course because this sort of aesthetic dentistry sings to my heart - aligning teeth offers conservative management of malocclusions with either no prep, or subsequently less prep, giving clients the opportunity to be the best of themselves.

'Dr Tif Qureshi was an excellent instructor and explained things very well. The format of the course was very good although we had a lot to fit into the allocated time; the hands-on part was very helpful.

'The Inman Aligner cases I have started have been a real hit with patients and there is a lot of interest and excitement over what these systems can offer. I have just bought a practice and we are now able to offer the Inman Aligner and ClearSmile Aligners. Further marketing will no doubt increase the number of cases I take on and further revolutionise the type of dentistry we can offer our patients.'

The Inman Aligner and ClearSmile Aligners are provided by the IAS Academy, and all training is led by leading professionals in the field of cosmetic orthodontics.

To find out more, contact the team today. For more information, go to www.iasortho.com, emailinfo@iasortho.com or call 08453665477.

\section{A NEW DENTURE ADVENTURE}

Wearing a partial denture almost doubles the patient's risk of losing more teeth than non partial wearers.

Tooth loss can have a profound effect on the lives of patients both physically and emotionally - 45\% of patients experience difficulties accepting the loss of their teeth. ${ }^{2}$

As their trusted dental professional, your advice can have a huge impact on a patient's wellbeing, whilst also helping them to avoid losing more teeth.

What's more, as the population ages, and the chance of wearing a denture increases with age, ${ }^{3}$ you will be seeing more denture patients on a day-to-day basis. Within the next 35 years, it is expected there will be 2 billion people aged $60+{ }^{4}$ Are you prepared for an increasing number of denture patients in your practice?

Support denture wearers to care for their denture and protect their oral health with the POLIGRIP Partial Wearer Starter Kit. Designed specifically for new partial denture wearers, the kits contain a range of materials to help them through each step of the denture journey.

The kit contains:

- A guide to starting life with partial dentures' - advice and support for patients considering a denture or requiring tooth extraction

- A flip chart designed to help you to explain the process to new denture patients, with helpful illustrations and graphs

- Discreet wash bags each containing advice for the first few days with a new denture, denture bath and brush, product samples and a money off coupon.

Request your FREE kits now while stocks last at www.gskdentalprofessionals.co.uk.

Preshaw P M et al. Association of removable partial denture use with oral and systemic health. J Dent 2011; 29: 711-719.

2. Davis D M et al. The emotional effects of tooth loss: a preliminary quantitative study. Br Dent J 2000; 188: 503-506.

3. Steele J G et al. Total tooth loss in the United Kingdom in 1998 and implications for the future. Br Dent J 2000; 189 598-603.

4. UNFPA \&t HelpAge International, 2012 